\title{
A Novel Optimization Framework for C-RAN BBU Selection based on Resiliency and Price
}

\author{
Mohammed Yazid Lyazidi*^, Lorenza Giupponi ${ }^{\star}$, Josep Mangues-Bafalluy ${ }^{\star}$, Nadjib Aitsaadi ${ }^{\dagger}$ and Rami Langar ${ }^{\ddagger}$ \\ ${ }^{*}$ Sorbonne Universities, UPMC Univ Paris 06, CNRS, LIP6 UMR 7606, 75005 Paris, France. \\ ${ }^{\star}$ Centre Tecnològic de Telecomunicacions de Catalunya (CTTC), 08860 Castelldefels, Barcelona, Spain. \\ ${ }^{\dagger}$ University Paris-Est, LIGM-CNRS UMR 8049, ESIEE Paris, 93162 Noisy-le-Grand, France. \\ ${ }_{\ddagger}^{\ddagger}$ University Paris-Est, LIGM-CNRS UMR 8049, UPEM, 77454 Marne-la-Valle, France. \\ Emails: yazid.lyazidi@lip6.fr, [lorenza.giupponi, josep.mangues]@cttc.es, nadjib.aitsaadi@esiee.fr, rami.langar@u-pem.fr
}

\begin{abstract}
As Mobile Network Operators (MNOs) are shifting towards Cloud-Radio Access Network (C-RAN), they have to upgrade their infrastructure to not only support higher processing capacities but also to be more resilient. We consider the problem where a MNO is faced with the choice of selecting virtualized Baseband Units (BBUs) from various cloud service providers, that are each characterized with distinct failure probabilities and prices. We propose to solve the BBU selection problem, formulated as an Integer Linear Program (ILP) subject to BBU capacity and virtualization cost using the Branch-andPrice algorithm. We present several schemes depicting which optimization goal the MNO can foster the most: BBU processing power minimization, resiliency, traffic handling or all. Simulation results demonstrate the good performance of our algorithm to solve the BBU selection problem for all schemes, while also emphasizing the advantages of a particular one that can realize more than $10 \%$ in virtualization cost savings.
\end{abstract}

Keywords: C-RAN, Optimization, BBU, Resiliency, Virtualization cost, Branch-and-Price

\section{INTRODUCTION AND RELATED WORKS}

Mobile Network Operators (MNOs) have recently shown keen eagerness to shift to Cloud Radio Access Network (C-RAN) solutions in order to improve their networks migration to LTE-Advanced Pro and meet the advent of the fifth generation (5G) [1][2]. While the premise of C-RAN and the technical feasibility of base station virtualization open up a window of attractive benefits in capital and operational expenses for MNOs, many challenges lie ahead before enabling C-RAN's full migration towards 5G and its commercial expansion. For instance, the optimization issues of latencies in fronthaul networks includes the study of functional splits and Remote Radio Heads (RRHs) deployment [3]. Other C-RAN related studies encompass end-users admission control regarding fronthaul links capacity and quality-of-service requirements [4]; Baseband Units (BBUs) virtualization [5]; development of dynamic resource sharing mechanisms [6]; and flexible BBU-RRH mappings [7].

However, it is unequivocal that very few research works have addressed the problem of resiliency in C-RAN along with the cost of instantiating the BBU pool. This constitutes a major requirement for MNOs to guarantee less limited disruptions in network availability throughout the day, while respecting the capacity budget and traffic load catering. Some related works have adumbrated this issue, such as [8], which proposed a partial cloud network mapping algorithm to lessen the failure disruptions with a cost on the available bandwidth. In [9], Bouet et al. proposed a placement scheme for virtualized BBUs in a cloud infrastructure to meet the operational cost constraints such as licence fees and/or power consumption. However, these proposals consist only of early work studies, which have not fully exploited the flexibility of centralized C-RAN architecture regarding efficient baseband resource pooling and its ability to benefit from cost-efficient optimization.

In this paper, we consider a C-RAN architecture where the $\mathrm{MNO}$ has to select BBU equipments from different Cloud Service Providers (CSPs) to run its virtualized baseband pool. Besides, we assume that each CSP's BBU is characterized by a failure probability [10] and a capacity cost, that can be equivalent to content delivery network prices [11] for the services required from CSPs. We propose in this context, a novel framework addressing the problem of optimal BBUs selection, from several CSPs, for a BBU-RRH traffic dependent C-RAN. The instantiated BBU pool should meet the MNO's expectations in terms of reliability, cost efficiency, processing power optimization and traffic load catering. To the best of our knowledge, this paper is the first attempt to present an optimization design for C-RAN BBU selection based on resiliency and virtualization price.

We formulate our selection problem, named Cost-Resilience BBU Selection (CRBS), as an Integer Linear Program (ILP) problem, designed as a weighted objective function with three optimization goals: i) minimizing the BBU pool processing power, ii) maximizing its resiliency and iii) increasing the RRHs traffic load handling, subject to virtualization's capacity and budget constraints. Additionally, we consider that each $\mathrm{RRH}$ is characterized by its hourly traffic, depending on the type of area it covers (e.g., business or residential area). It is worth noting that the third optimization goal focuses on maximizing the overall percentage of traffic that can be handled from the lowest traffic load RRHs at a given hour, subject to ensuring the management of the high-load ones. In fact, we consider that the traffic from the highest traffic load RRHs will not only generate a highest average traffic volume, but also highest peaks. Therefore, since there are more RRHs distributed throughout the radio site, such RRHs 
will be closer to the end user, and thus, will be able to cater best to the user's capacity demand. We also assume that an overlaying macro cell will handle the remaining traffic from lowest traffic load RRHs if the BBU pool's capacity is reached at that specific hour. To solve the ILP CRBS problem, we propose to employ the Branch-and-Price (B\&P) algorithm [12], which is a combination of the Branch-and-Bound and Column Generation methods for efficiently solving large-scale ILP problems.

The remainder of the paper is organized as follows: Section II presents the mathematical formulation of our system model. In section III, we present our algorithm design to solve the CRBS problem. Performance evaluation of our proposal is discussed in section IV, followed by Section $\mathrm{V}$, which concludes the paper.

\section{System Model And Problem Formulation}

We consider a two-tier C-RAN architecture composed of a macro cell, overlaying a number of $S$ RRHs [13]. We denote by $N$ the number of BBU candidates that the $\mathrm{MNO}$ is inclined to instantiate in its $\mathrm{BBU}$ pool $\mathcal{B}=\{i \mid 1 \leq i \leq N\}$ to handle the traffic load of all $S$ RRHs. The number $N$ of needed BBUs can be deduced from the overall existent traffic load on all RRHs and the downlink capacity of the operator, as has been highlighted in [7]. Each CSP's BBU candidate $i$ is characterized by its per Mbps pricing $m_{i}$ and its failure probability $p_{i}$. We denote by $r_{i j}$ the binary variable, which is equal to 1 if $\mathrm{BBU} i$ handles $\mathrm{RRH} j$, and 0 otherwise. Without loss of generality, we assume that each BBU is limited by a fixed capacity $C$ in terms of traffic it can handle [5]. We define the average utilization of a BBU $i$ as follows:

$$
y_{i}=\sum_{j=1}^{S} r_{i j} l_{j} / C
$$

where $l_{j}$ is the current traffic in RRH $j$. The BBU-RRH dependent traffic is realized by a functional split separating user and cell related functions [3]. Besides, we consider that the baseband processing power consumption in a single BBU is linear with its average utilization. Thus, the processing power $P_{i}$ consumed at BBU $i$ can be expressed as:

$$
P_{i}=P_{0}+\delta P_{\max } y_{i}
$$

In particular, $P_{0}$ represents the power in the BBU when the latter is in idle mode, and $P_{\max }$ when in full usage mode. $\delta$ is a constant between 0 and 1 , which represents the slope of the equivalent linear power model. On the other hand, we suppose that the failure probability of the $\mathrm{BBU}$ pool $p(\mathcal{B})$ is equal to 1 if $\mathcal{B}=\emptyset$, and $\prod_{i} p_{i}$, otherwise. In order to optimize this term as one of the MNO's intended goals for enhancing the cloud's resiliency, we transform the product of probabilities into linear summation by defining the following function $I_{i}$, which is equal to the negative value of the logarithmic function on the failure probability of BBU $i$, i.e. $I_{i}=-\log \left(p_{i}\right)$. Consequently, we can write:

$$
I_{\mathcal{B}}=-\log (p(\mathcal{B}))=-\sum_{i} \log \left(p_{i}\right)=\sum_{i} I_{i}
$$

Furthermore, we consider two sets of RRHs in the deployed architecture denoted as $\mathcal{S}_{\mathcal{H}}$ and $\mathcal{S}_{\mathcal{L}}$, which represent the sets of RRHs with high (maximum) traffic load and low (minimum) traffic load, respectively.

We formulate in the following our mathematical CRBS optimization problem $(\mathcal{P})$, expressed as a generic weighted optimization problem with three homogenised objective terms:

$$
\begin{aligned}
\underset{r}{\operatorname{minimize}} \quad & \alpha \sum_{i=1}^{N} \frac{x_{i} P_{0}+\delta . P_{\max } y_{i}}{P_{\max }}-\beta \sum_{i=1}^{N} x_{i} \frac{I_{i}}{\max (I)} \\
& -\gamma \frac{\sum_{i=1}^{N} \sum_{j \in \mathcal{S}_{\mathcal{L}}} r_{i j} l_{j}}{\sum_{j \in \mathcal{S}_{\mathcal{L}}} l_{j}} \\
\text { subject to : } \quad & \sum_{j=1}^{S} r_{i j} l_{j} \leq C, \forall i \\
& \sum_{i=1}^{N} r_{i j} \leq 1, \forall j \\
& \sum_{i=1}^{N} m_{i} \sum_{j=1}^{S} r_{i j} l_{j} \leq B \\
& \sum_{i=1}^{N} \sum_{j \in \mathcal{S}_{\mathcal{H}}} r_{i j} l_{j}=\sum_{j \in \mathcal{S}_{\mathcal{H}}} l_{j} \\
& r_{i j} \in\{0,1\}, \forall i, j
\end{aligned}
$$

The proposed objective function consists to minimize the total BBU pool processing power, while maximizing the resiliency (or minimizing the failure probability) of the pool as well as the traffic load that can be handled from the low-traffic RRHs by the instantiated BBUs. $\max (I)$ represents the maximum value of $\left\{I_{1}, \ldots, I_{N}\right\}$. We define $\alpha, \beta$ and $\gamma$ as constant weights between 0 and 1 , and whose total sum is equal to 1 (i.e., $\alpha+\beta+$ $\gamma=1$ ). We consider that these constants are set in advance by the MNO in order to fix the optimization strategy depending on its most prevailing focuses. We define a binary indicator $x_{i}$, which represents the statue of BBU $i,\left(x_{i}=0\right.$, if $\sum_{j=1}^{S} r_{i j}=$ 0 , i.e., BBU $i$ is inactive, and 1 otherwise). $(C 1)$ is the total BBU resource usage limitation constraint, and constraint $(C 2)$ implies that a RRH cannot be shared by more than one BBU. Meanwhile, constraint $(C 3)$ denotes that the capacity costs of all instantiated BBUs should be less than or equal to the MNO's virtualization budget $B$. Also, constraint $(C 4)$ ensures that the traffic of high-load RRHs is $100 \%$ handled and, finally, $(C 5)$ indicates that variable $r_{i j}$ is binary.

\section{Proposed B\&P Algorithm Design for Solving THE CRBS PROBLEM}

The CRBS problem formalized in $(\mathcal{P})$ is ILP and cannot be solved directly using convex optimization techniques. Besides, problem $(\mathcal{P})$ is NP-hard [5] and can only be solved by exhaustively figuring out all $N^{S}$ possible combinations of the BBU-RRH assignment variable, which is impracticable for large-scale networks. To find solutions to our problem, we propose to make use of the $\mathrm{B} \& \mathrm{P}$ framework, which combines the branch-and-bound and column generation approaches to 
compute the optimal solution of ILP problems [12]. The algorithm is based on solving by column generation the linear relaxation in each node of a branch-and-bound tree. In the B\&P algorithm, a sets of columns are left out of the LP relaxation in order to handle the problem more efficiently by decreasing the computational difficulty. Columns are then "priced" and added back to the LP relaxation as needed. To decide which column will be added, a sub-problem called the "pricing problem" is created to identify which columns should enter the basis so as to increase the objective function (in case of maximization problem). If such columns are found, the LP is then re-optimized. We detail next, the steps of designing each of the Master and Pricing problems for our B\&P algorithm.

The first step consists in reformulating the original problem by applying the well-known Dantzig-Wolfe's reformulation [12], that sub-divides the problem into a Master and a Pricing Problem. However, before applying the problem reformulation, we found that it is convenient to transform problem $(\mathcal{P})$ by considering two binary variables $v$ and $w$ instead of single variable $r$, which represent low and high-traffic load RRHs assignments to BBUs, respectively. The new CRBS problem $\left(\mathcal{P}^{\prime}\right)$ can be expressed as follows:

$$
\begin{array}{ll}
\underset{v, w}{\operatorname{maximize}} & \sum_{i=1}^{N} \Phi_{i} x_{i}+\Psi \sum_{i=1}^{N} \sum_{j \in \mathcal{S}_{\mathcal{H}}} w_{i j} l_{j}^{H} \\
& +\Omega \sum_{i=1}^{N} \sum_{j \in \mathcal{S}_{\mathcal{L}}} v_{i j} l_{j}^{L}
\end{array}
$$

$$
\text { subject to : }
$$$$
\sum_{j \in \mathcal{S}_{\mathcal{H}}} w_{i j} l_{j}^{H}+\sum_{j \in \mathcal{S}_{\mathcal{L}}} v_{i j} l_{j}^{L} \leq C, \forall i
$$$$
\sum_{i=1}^{N} v_{i j} \leq 1, \forall j \in \mathcal{S}_{\mathcal{L}}
$$$$
\sum_{i=1}^{N} w_{i j} \leq 1, \forall j \in \mathcal{S}_{\mathcal{H}}
$$$$
\sum_{i=1}^{N} m_{i}\left(\sum_{j \in \mathcal{S}_{\mathcal{H}}} w_{i j} l_{j}^{H}+\sum_{j \in \mathcal{S}_{\mathcal{L}}} v_{i j} l_{j}^{L}\right) \leq B
$$$$
\sum_{i=1}^{N} \sum_{j \in \mathcal{S}_{\mathcal{H}}} w_{i j} l_{j}^{H}=\sum_{j \in \mathcal{S}_{\mathcal{H}}} l_{j}^{H}
$$$$
v_{i j}, w_{i j} \in\{0,1\}, \forall i, j
$$

where: $\Phi_{i}=\beta I_{i} / \max (I)-\alpha P_{0} / P_{\max } ; \Psi=-\alpha \delta / C ; \Omega=$ $\gamma / \sum_{j \in \mathcal{S}_{\mathcal{L}}} l_{j}^{L}-\alpha \delta / C$. We denote by $l_{j}^{L}$ and $l_{j}^{H}$ the traffic in low and high-traffic load RRH $j$, respectively. Also, $x_{i}=0$, if $\sum_{j \in \mathcal{S}_{\mathcal{H}}} w_{i j}+\sum_{j \in \mathcal{S}_{\mathcal{L}}} v_{i j}=0$, and 1 otherwise. We apply next Dantzig-Wolfe's reformulation. Let $\mathcal{K}_{i}^{L}=\left\{v_{1}^{i}, v_{2}^{i}, \ldots, v_{k_{i}}^{i}\right\}$ and $\mathcal{K}_{i}^{H}=\left\{w_{1}^{i}, w_{2}^{i}, \ldots, w_{k_{i}}^{i}\right\}$ be the sets of possible feasible assignments of low and high-traffic load RRHs to BBU $i$, respectively. In this case, $v_{k}^{i}=\left\{v_{1 k}^{i}, v_{2 k}^{i}, \ldots, v_{S k}^{i}\right\}$ and $w_{k}^{i}=$ $\left\{w_{1 k}^{i}, w_{2 k}^{i}, \ldots, w_{S k}^{i}\right\}$ constitute a feasible solution to problem $\left(\mathcal{P}^{\prime}\right)$. Let $z_{k}^{i}=\left(\dot{z}_{k}^{i}, \ddot{z}_{k}^{i}\right)$ be a new variable, which is equal to $(1,1)$ if feasible solution $\left(v_{k}^{i}, w_{k}^{i}\right)$ is selected, and $(0,0)$ otherwise. We express in the following the Master Problem:

$$
\begin{aligned}
\underset{z}{\operatorname{maximize}} \quad & \sum_{i=1}^{N} \sum_{k=1}^{k_{i}}\left(\Phi_{i} x_{i}+\Psi \sum_{j \in \mathcal{S}_{\mathcal{H}}}\left(w_{j k}^{i} l_{j}^{H}\right) \ddot{z}_{k}^{i}\right. \\
& \left.+\Omega \sum_{j \in \mathcal{S}_{\mathcal{L}}}\left(v_{j k}^{i} l_{j}^{L}\right) \dot{z}_{k}^{i}\right) \\
\text { subject to : } & \sum_{i=1}^{N} \sum_{k=1}^{k_{i}} v_{j k}^{i} \dot{z}_{k}^{i} \leq 1, \forall j \in \mathcal{S}_{\mathcal{L}} \\
& \sum_{i=1}^{N} \sum_{k=1}^{k_{i}} w_{j k}^{i} \ddot{z}_{k}^{i} \leq 1, \forall j \in \mathcal{S}_{\mathcal{H}} \\
& \sum_{k=1}^{k_{i}} \dot{z}_{k}^{i} \leq 1, \sum_{k=1}^{k_{i}} \ddot{z}_{k}^{i} \leq 1, \forall i \\
& \sum_{i=1}^{N} m_{i} \sum_{k=1}^{k_{i}}\left(\sum_{j \in \mathcal{S}_{\mathcal{H}}} w_{j k}^{i} \ddot{z}_{k}^{i} l_{j}^{H}+\sum_{j \in \mathcal{S}_{\mathcal{L}}} v_{j k}^{i} \dot{z}_{k}^{i} l_{j}^{L}\right) \leq B \\
& \sum_{i=1}^{N} \sum_{k=1}^{k_{i}} \sum_{j \in \mathcal{S}_{\mathcal{H}}} w_{j k}^{i} \ddot{z}_{k}^{i} l_{j}^{H}=\sum_{j \in \mathcal{S}_{\mathcal{H}}} l_{j}^{H} \\
& \dot{z}_{k}^{i}, \ddot{z}_{k}^{i} \in\{0,1\}, \forall i, k
\end{aligned}
$$

In the Master Problem $(\mathcal{M P}), z_{k}^{i}$ represents a feasible assignment of RRHs to BBU $j$. Note that $(\mathcal{M P})$ cannot be solved directly due to its exponential number of columns, this is why we consider only a subset of columns that constitutes the Restricted Master Problem (RMP), where the values of the variables that do not appear are padded to zero. The observation is, for large-scale ILPs, most columns will have their associated variables equal to zero in any optimal solution anyway. Let $z^{\star}$ be the corresponding dual solution of the RMP. The next step consists in adding a number of columns with positive reduced cost that are found by solving the two following sub-problems:

$$
\underset{1 \leq i \leq N}{\operatorname{maximize}} \quad\left\{u^{i}-z^{\star i}\right\}
$$

where $u^{i}=\left(\dot{u}^{i}, \ddot{u}^{i}\right)$ is the optimal solution of the following Pricing Problem $(\mathcal{P P})$ :

$$
\begin{array}{ll}
\underset{v^{i}, w^{i}}{\operatorname{maximize}} & \Phi_{i} x_{i}+\Psi \sum_{j \in \mathcal{S}_{\mathcal{H}}}\left(l_{j}^{H}-w_{j}^{\star}\right) w_{j}^{i} \\
& +\Omega \sum_{j \in \mathcal{S}_{\mathcal{L}}}\left(l_{j}^{L}-v_{j}^{\star}\right) v_{j}^{i} \\
\text { subject to : } & \sum_{j \in \mathcal{S}_{\mathcal{H}}} w_{j}^{i} l_{j}^{H}+\sum_{j \in \mathcal{S}_{\mathcal{L}}} v_{j}^{i} l_{j}^{L} \leq C \\
& v_{i j}, w_{i j} \in\{0,1\}, \forall j
\end{array}
$$

where $v_{j}^{\star}$ and $w_{j}^{\star}$ correspond to the optimal dual price from the solution of the RMP associated with the partitioning constraints of low and high-traffic load RRH $j$, respectively. In the Pricing Problem $(\mathcal{P} \mathcal{P})$, we generate the best feasible low and high-traffic load RRH assignments from all the feasible 




Fig. 1: B\&P algorithm Flow Chart

Table I: Optimization strategies

\begin{tabular}{|c|c|}
\hline Scheme & $(\alpha, \beta, \gamma)$ \\
\hline Total Power Minimization Scheme (TPMiS) & $(1 ; 0 ; 0)$ \\
\hline Resilience Maximization Scheme (RMaS) & $(0 ; 1 ; 0)$ \\
\hline LP Traffic Maximization Scheme (LTMaS) & $(0 ; 0 ; 1)$ \\
\hline Equal-Weighted Optimization Scheme (EWOS) & $(1 / 3 ; 1 / 3 ; 1 / 3)$ \\
\hline 424-Scheme (424-S) & $(0.4 ; 0.2 ; 0.4)$ \\
\hline
\end{tabular}

Table II: CSP inputs from [10][11]

\begin{tabular}{|c|c|c|c|}
\hline CSP-i & Failure probab. $p_{i}$ & $I_{i}$ & pricing $m_{i}$ \\
\hline CSP-1 & 0.01 & 2 & $0.8 \$ / M b p s$ \\
\hline CSP-2 & 0.05 & 1.30103 & $0.9 \$ / M b p s$ \\
\hline CSP-3 & 0.1 & 1 & $1 \$ / M b p s$ \\
\hline CSP-4 & 0.01 & 2 & $1.1 \$ / M b p s$ \\
\hline
\end{tabular}

ones for each BBU $i$. After that, we look for the best BBU$\mathrm{RRH}$ assignments over all BBUs, which is precisely done by problem (16). Fig. 1 summarizes the B\&P algorithm's flowchart. It is worth noting that branching in the B\&P occurs when no columns have been "priced out" to enter the basis and the LP solution does not satisfy constraints. Furthermore, it is not required to solve $(\mathcal{P P})$ to optimality; in fact, any column with a positive reduced cost can be accepted. Hence, if the value of the objective function to the column generation sub-problem is less or equal to zero, then the current optimal solution for the RMP is also optimal for $(\mathcal{M P})$.

\section{Performance Evaluation}

In this section, we evaluate the benefits of the $\mathrm{B} \& \mathrm{P}$ algorithm to solve the CRBS problem, while comparing the results for different scenarios of the optimization weights $(\alpha, \beta, \gamma)$. We have tested different combinations and outlined in Table I the most representative of the other weights values. The 424$\mathrm{S}$ was chosen in consideration that reliability may be twice

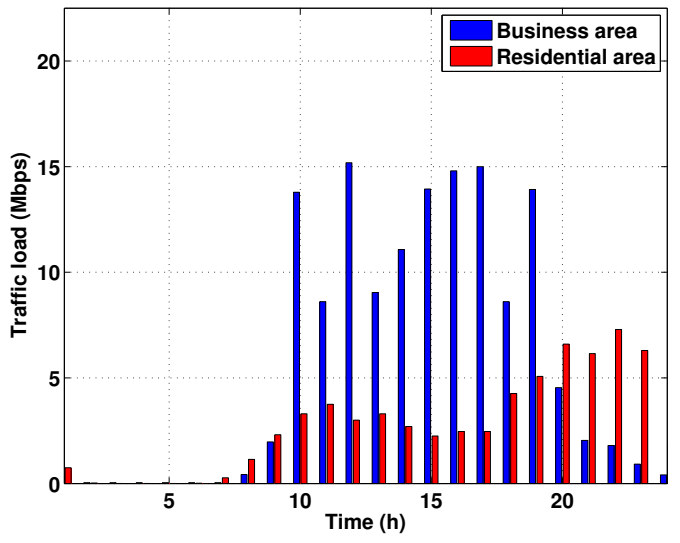

Fig. 2: Daily traffic load observed on business and residential area RRHs during a workday [1].

Table III: Simulation Parameters

\begin{tabular}{|c|c|}
\hline Parameters & Values \\
\hline Number of RRHs $S$ & 19 \\
\hline Number of business RRHs & 15 \\
\hline Number of residential RRHs & $\in[1 ; 19]$ (default 4$)$ \\
\hline$P_{0}(W) / \delta / P_{\max }(W)$ & $1.25 / 1 / 3.75$ \\
\hline Number of BBUs $N$ & $8(2$ from each CSP) \\
\hline BBU capacity $C(M b p s)[3]$ & 200 \\
\hline Bandwidth & $10 \mathrm{MHz}$ \\
\hline Physical Resource Blocks & 50 \\
\hline Virtualization budget $B[11]$ & $230 \$ /$ hour $(2 \mathrm{M} \$ /$ year $)$ \\
\hline
\end{tabular}

less important for a MNO than to serve the whole traffic and minimize the total C-RAN BBU processing power. In all our simulation scenarios, we consider four CSPs, with their corresponding failure probabilities, $I$-function values and price per Mbps that are detailed in Table II (data of existent commercial content delivery network that can be closely equivalent to CSPs can be found in [11]). The rest of the default simulation parameters are described in Table III. We consider a total of $S=19$ RRHs, with 15 business and 4 residential RRHs, differentiated by an hourly traffic load given by [1] and illustrated in Fig. 3. The observation is the number of RRHs that can be within the low traffic set $\mathcal{S}_{\mathcal{L}}$ or the highest one $\mathcal{S}_{\mathcal{H}}$ varies depending on the hour. We use the commercial solver IBM ILOG CPLEX [14] to solve both the LP relaxation of the RMP and the Pricing Problem. The average overall computation time of the B\&P algorithm is less than $6.5 \mathrm{~ms}$. For the sake of comparison to a static benchmark, we compare the results to a Static Selection Scheme (SSS) where the MNO targets to satisfy maximum achieved network load at all hours, so as to ensure maximum users quality of service, while contracting with only one CSP (CSP-4) and with no budget constraint. In what follows, we present our performance metrics in terms of: i) BBU pool processing power, ii) resiliency, iii) number of active RRHs, iv) virtualization cost and v) the evolution of the percentage of handled traffic load while varying the number of residential RRHs in the network. 


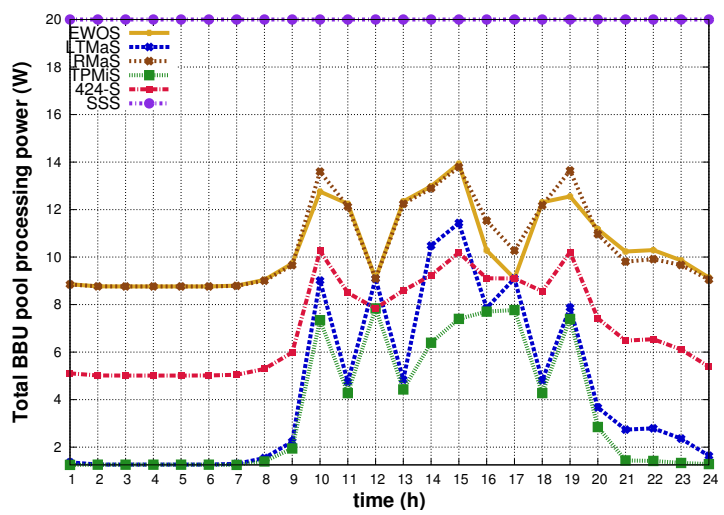

Fig. 3: BBU pool processing power vs time

Table IV: Average BBU pool failure probability and number of BBUs

\begin{tabular}{|c|c|c|c|c|c|}
\hline Scheme & TPMiS & RMaS & LTMaS & EWOS & $424-\mathrm{S}$ \\
\hline$p(\mathcal{B})$ & $\leq 10^{-2}$ & $\leq 5.10^{-6}$ & $\leq 10^{-2}$ & $\leq 5.10^{-6}$ & $\leq 10^{-4}$ \\
\hline Min. & 1 & 3 & 1 & 3 & 2 \\
\hline Max. & 2 & 8 & 5 & 7 & 4 \\
\hline
\end{tabular}

\section{A. BBU pool processing power}

We compare in Fig. 4 the hourly BBU pool processing power returned from the six approaches. We can remark an adaptive behaviour to the fluctuating traffic load for all weights scenarios schemes, with TPMiS having the minimum power consumption. In fact, the latter instantiates the least number of BBUs compared to the others, which lessens the total BBU pool processing power. LTMaS comes second in power minimization, since it has to handle more traffic coming from low traffic load RRHs. Besides, we can remark that 424-S consumes less processing power than LTMaS at certain peak traffic hours, such as at $h=12: 00, h=14: 00$ and $h=15: 00$. On the other hand, RMaS has the second highest power consumption, since it tends to maximize the number of invoked BBUs to increase the resiliency. Furthermore, we measured that EWOS and 424-S consume at maximum $30 \%$ and $37.5 \%$ less processing power than SSS, respectively.

\section{B. Resiliency}

Table IV presents the different values of the BBU pool's highest failure probability $p(\mathcal{B})$ during the day, as well as the minimum and maximum number of instantiated BBUs. Since $p(\mathcal{B})$ is the product of all invoked BBUs failure probabilities, the more BBUs are instantiated, the smaller is the failure probability and the more resilient is the BBU pool. This can be seen in both the RMaS and EWOS schemes as they achieve the maximum resiliency throughout the day by invoking at least more than three BBUs from CSP-2 and CSP-1 and/or CSP-3. On the other hand, TPMiS and LTMaS usually start with few number and BBUs, then instantiates as many as possible to accommodate to peak traffic at high-traffic load hours, and the extra coming from low traffic load RRHs for LTMaS. Regarding 424-S, it invokes fewer number of BBUs than EWOS but, as will be seen next, can handle more RRH traffic.

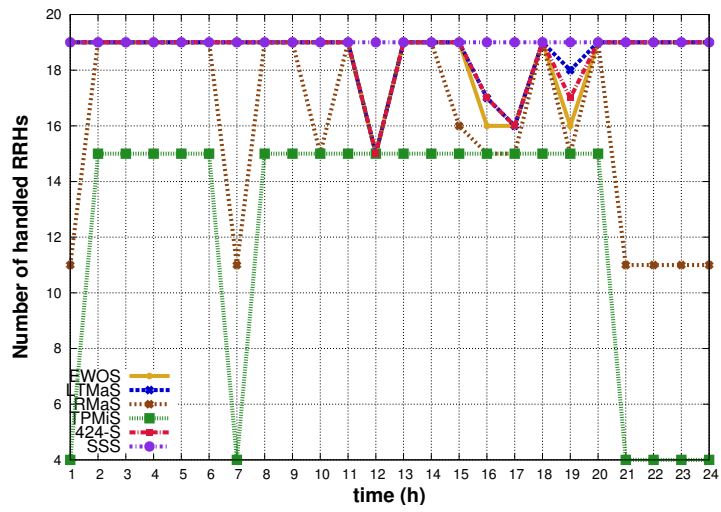

Fig. 4: Number of active RRHs vs time

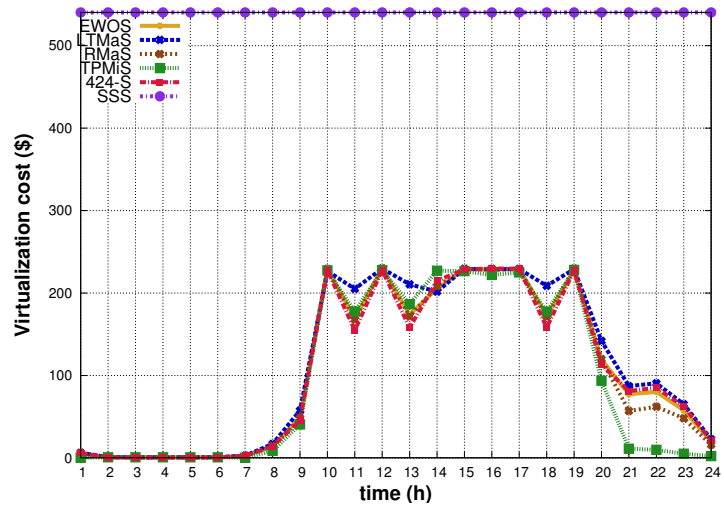

Fig. 5: Virtualization cost vs time

\section{Number of active RRHs}

The evolution of the number of active RRHs is depicted in Fig. 5, where it is shown that EWOS, 424-S and LTMaS maximize the total number of handled RRHs. As for TPMiS and RMaS, they cater exclusively to the load of high-traffic cells, since they tend to minimize and maximize, respectively, the number of BBUs $(\gamma=0)$. However, we remark that at peak traffic hours such as at $h=12: 00, h=16: 00, h=17: 00$, and $h=19: 00$, not all RRHs could be handled by EWOS, 424-S and LTMaS, unlike SSS, due to restricted BBU capacity and budget (as will be detailled in the next figure). Meaning that, all (or a major part) of the traffic from residential areas during office hours will be handled by the macro base station. We can also note how 424-S performs better than EWOS at $h=16: 00$ and $h=19: 00$ as it handles more RRHs traffic.

\section{Virtualization cost}

Table V: MNO annual expenditure and cost savings

\begin{tabular}{|c|c|c|c|c|c|}
\hline Scheme & TPMiS & RMaS & LTMaS & EWOS & $424-S$ \\
\hline Total cost (K\$) & 1,681 & 1,775 & 1,968 & 1,843 & 1,820 \\
\hline Annual savings & $16 \%$ & $11.3 \%$ & $1.6 \%$ & $7.9 \%$ & $9.8 \%$ \\
\hline Savings to SSS & $64.5 \%$ & $62.5 \%$ & $58.5 \%$ & $62.1 \%$ & $63 \%$ \\
\hline
\end{tabular}

The study of the virtualization cost is presented in Fig. 6 with the annual expenditure and cost saving to both the annual 


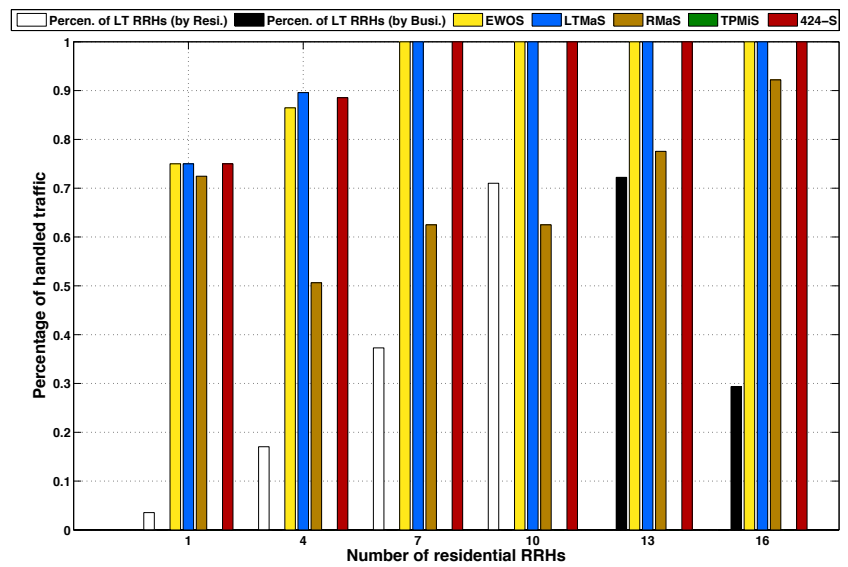

Fig. 6: Average percentage of handled low traffic RRH vs number of residential cells

budget and SSS in Table V. We can remark that at peak traffic hours, the MNO's budget limitation $B$ of $230 \$$ per hour is reached for most schemes, with TPMiS realizing minimum cost from $h=20: 00$ to $h=09: 00$ and 424-S having the lowest cost at peak traffic hours. LTMaS on the other hand is the most costly with only $1.6 \%$ of savings of the total annual budget and $58.5 \%$ to SSS. Consequently, to allow more traffic catering, the MNO should either boost its budget limit to reach the SSS benchmark; or extend its contractual budget with more CSPs to cater to more traffic; or increase the BBU capacity $C$, with the repercussion of decreasing its total cost savings. A special mention goes to the 424-S approach, which achieves almost $10 \%$ of annual savings and $63 \%$ to the static approach. Besides, 424-S is not only more cost-efficient than EWOS, but, as was seen previously, instantiates less BBUs to cater to the same, if not more, RRHs loads throughout the day.

\section{E. Percentage of handled low traffic load}

In Fig. 7, we study the evolution of the average percentage of handled low-traffic RRHs, while varying the number of residential RRHs in the network. The average is taken from the 24 values of the day. With the increase of the number of residential RRHs, the load of the business ones become less important in the network and then switches to become the set $\mathcal{S}_{\mathcal{L}}$. This allows the BBU pool to handle $100 \%$ of all the RRHs load by EWOS, LTMaS and 424-S. Besides, since RMaS tends only to maximize the number of BBUs while satisfying constraint $(C 4)$, it consequently increases the capacity of the BBU pool to handle extra traffic from the residential cells, but still not achieving $100 \%$. We can note the absence of TPMiS since $\gamma=0$.

\section{F. Final Remarks}

From our analysis, we can deduce that decreasing the resiliency's weight for selecting BBUs in favor of the processing power and traffic handling can yield the best results. This was assessed in the 424-S case which outperformed the tradeoff scheme EWOS. In fact, 424-S proved to be a good strategy choice for MNOs to satisfy $100 \%$ of traffic with the minimum number of BBUs, provided they can tolerate its failure probability of $10^{-4}$ (which is faultless) and meet the total expenditure of the scheme.

\section{CONClusion}

In this paper, we have evaluated several BBU selection policies and provided general guidelines for MNOs to decide the best optimization strategy according to their needs: BBU processing power minimization, resiliency, traffic handling or all. More precisely, the presented EWOS and 424-S approaches have shown their ability to adapt to most traffic load scenarios and answer MNOs' major constraints. Especially, the 424-S may be the best option if a MNO targets to instantiate $50 \%$ less BBUs, and realize $37.5 \%$ of BBU power savings and $10 \%$ of virtualization expenditure economy.

\section{ACKNOWLEDGMENT}

This work has been partially funded by European EIT Digital program, the Spanish Ministry of Economy and Competitiveness under grant TEC2014-60491-R (5GNORM) and the FUI ELASTIC Network project (Grant no. C16/0287).

\section{REFERENCES}

[1] C-RAN, The Road Towards Green RAN, China Mobile Research Institute, White Paper, Version 3.0, December 2013.

[2] Nokia, "Multi-layer and cloud-ready radio evolution towards 5G, 2016 , white paper, available online at: https://gsacom.com/paper/multi-layercloud-ready-radio-evolution-towards-5g," 2016.

[3] A. Checko, A. P. Avramova, M. S. Berger, and H. L. Christiansen, "Evaluating c-ran fronthaul functional splits in terms of network level energy and cost savings," Journal of Communications and Networks, vol. 18, no. 2, pp. 162-172, April 2016.

[4] M. Y. Lyazidi, N. Aitsaadi, and R. Langar, "Resource allocation and admission control in OFDMA-based cloud-RAN," in 2016 IEEE Global Communications Conference (GLOBECOM), Dec 2016, pp. 1-6.

[5] M. Qian, W. Hardjawana, J. Shi, and B. Vucetic, "Baseband processing units virtualization for cloud radio access networks," IEEE Wireless Communications Letters, vol. 4, no. 2, pp. 189-192, April 2015.

[6] B. Niu, Y. Zhou, H. Shah-Mansouri, and V. W. S. Wong, "A dynamic resource sharing mechanism for cloud radio access networks," IEEE Transactions on Wireless Communications, vol. 15, no. 12, pp. 83258338, Dec 2016.

[7] M. Y. Lyazidi, N. Aitsaadi, and R. Langar, "Dynamic resource allocation for cloud-RAN in LTE with real-time BBU/RRH assignment," in 2016 IEEE International Conference on Communications (ICC), 2016, pp. $1-6$.

[8] C. Colman-Meixner, G. B. Figueiredo, M. Fiorani, M. Tornatore, and B. Mukherjee, "Resilient cloud network mapping with virtualized BBU placement for Cloud RAN," 10th IEEE International Conference on Advanced Networks and Telecommunications Systems IEEE ANTS, 2016.

[9] M. Bouet, J. Leguay, and V. Conan, "Cost-based placement of vdpi functions in nfv infrastructures," in Proceedings of the 2015 1st IEEE Conference on Network Softwarization (NetSoft), April 2015, pp. 1-9.

[10] " $100 \%$ availability for cloud and data center applications. [online] available: http://www.cedexis.com/."

[11] D. Rayburn, "Current state of the CDN market: DIY, pricing trends, competitive dynamics, may 2016, available online at: www.cdnpricing.com," 2016.

[12] C. Barnhart, E. Johnson, G. Nemhauser, M. Savelsbergh, and P. Vance, "Branch-and-price: Column generation for solving huge integer programs," Oper. Res., pp. 46(3):316-329, 1998.

[13] A. Abdelnasser and E. Hossain, "Resource allocation for an OFDMA cloud-RAN of small cells underlaying a macrocell," IEEE Transactions on Mobile Computing, vol. 15, no. 11, pp. 2837-2850, 2016.

[14] "IBM ILOG CPLEX Optimizer Version 1 v12.6. available: http://www01.ibm.com/software/commerce/optimization/cplex-optimizer/." 